\title{
USO DE BALÃO INTRA-HEPÁTICO PARA CONTROLE TEMPORÁRIO DA HEMORRAGIA EM LESÃO DA VEIA CAVA RETRO-HEPÁTICA
}

\author{
INTRAHEPATIC BALLOON TAMPONADE FOR TEMPORARY CONTROL OF \\ RETROHEPATIC VENA CAVA INJURY BLEEDING
}

\author{
João Eduardo Leal Nicoluzzi, TCBC-PR ${ }^{1}$; Marlon Rangel,ACBCPR ${ }^{2}$; \\ Luís Carlos VonBahten,TCBC - PR
}

\section{INTRODUÇÃO}

As lesões de vasos justa-hepáticos, veias hepáticas ou porção retro hepática da veia cava (RVC), assim como da porção intra-hepática dos ramos venosos principais, são injúrias associadas a elevados índices de mortalidade. Os relatos isolados de pacientes que sobreviveram a este tipo de lesão foram na maioria manejado por técnicas de isolamento vascular do fígado, normalmente associado a shunt átrio-caval (SAC). A alta mortalidade deste tipo de lesão devese a múltiplos fatores, incluindo demora no diagnóstico, falta de familiaridade com a técnica de SAC e uso do shunt quando as demais técnicas falharam ${ }^{1}$. O SAC apresenta mortalidade acima de $90 \%$ e outras manobras geralmente falham devido à hemorragia profusa associada à lesão de veias hepáticas ${ }^{2}$.

A alta mortalidade deste tipo de lesão tem sido atribuída principalmente ao difícil acesso à porção retro-hepática da veia cava inferior, a qual se localiza atrás da porção mais espessa e menos móvel do fígado ${ }^{3}$. Esta disposição anatômica acarreta grande dificuldade para exposição e controle direto de hemorragia traumática proveniente deste vaso. Qualquer tentativa de rotação do fígado para exposição tende a aumentar a hemorragia e apresenta risco adicional de embolia gasosa.

Neste artigo apresenta-se um caso inédito de paciente com lesão de RVC por arma de fogo, com hemorragia controlada pela inserção de balão intra-hepático após o insucesso de outras manobras. $\mathrm{O}$ balão permaneceu insuflado por dois dias e os princípios do damaged control e reoperação planejada foram empregados neste doente ${ }^{4,5}$.

\section{RELATO DO CASO}

Um paciente do sexo masculino, 23 anos, com lesão por arma de fogo no quadrante superior direito, sem orifício de saída, foi recebido no Serviço de Emergência 25 minutos após o trauma, hipotenso (pressão arterial sistólica $<60$ mm
Hg) com abdômen distendido e doloroso à palpação. Submetido à laparotomia observou-se lesão estrelada na parte superior do fígado direito entre os segmentos IV e VIII. A mobilização hepática acarretou hemorragia profusa optamos então pela insuflação de um balão intra-hepático para tentar controlar o sangramento (Figura 1) e pelo fechamento da incisão opera-

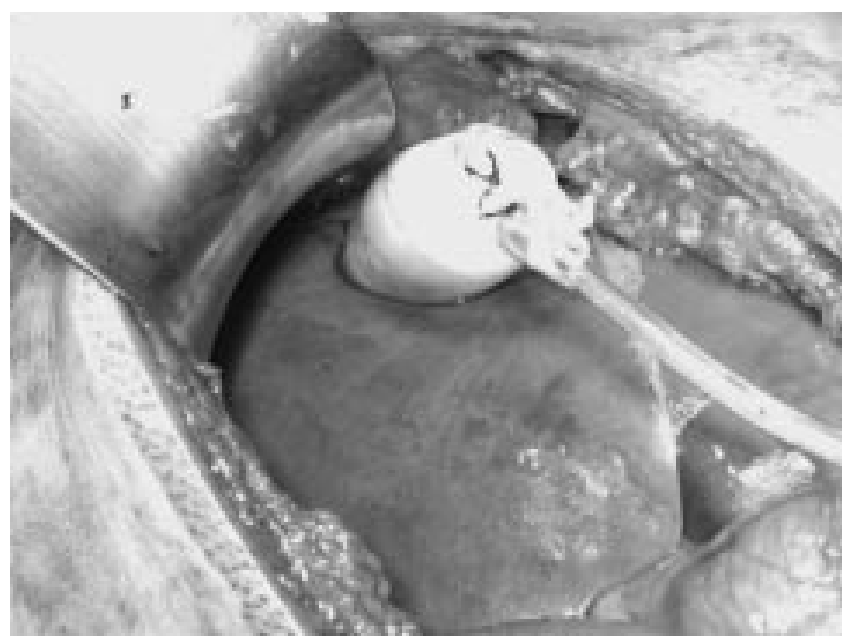

Figura 1 - Balão Intra-hepático no interior da lesão.

tória para correção da hipotermia e distúrbios metabólicos para posterior reoperação programada. $\mathrm{O}$ paciente foi então transferido para unidade de terapia intensiva para continuidade do tratamento. Após dois dias o paciente apresentavase estável hemodinamicamente, com boa diurese e normotérmico. Decidiu-se então pela reoperação para tentarse uma exclusão vascular do fígado e correção da lesão. Os clampes vasculares foram então sequencialmente posicionados no pedículo hepático, veia cava infra-hepática e veia cava supra-hepática. Como o doente não apresentou qualquer instabilidade o balão foi removido e o fígado liberado da veia cava retro-hepática. Após esta manobra observamos uma lesão transfixante na veia cava, logo abaixo dos

\footnotetext{
1. Professor Adjunto de Cirurgia do Serviço de Transplante Hepático e Pancreático do Hospital Angelina Caron; Mestre e Doutor em Cirurgia; Professor Adjunto da Pós-Graduação de Cirurgia da PUC-PR; Cirurgião do Hospital Universitário Cajuru-PUC-PR.

2. Cirurgião do Serviço de Cirurgia Geral e do Trauma do Hospital Universitário Cajuru da PUC-PR.

3. Professor Titular de Cirurgia da PUC-PR, Mestre e Doutor em Cirurgia. Cirurgião do Hospital Universitário Cajuru-PUC-PR.

Recebido em 09/09/2005
}

Aceito para publicação em 18/11/2005

Conflito de interesses: nenhum

Fonte de financiamento: nenhuma

Trabalho realizado no Serviço de Cirurgia - Geral e do Trauma do Hospital Universitário Cajurú da PUC-PR, Curitiba PR. 
vasos supra-hepáticos (Figura 2). A lesão foi então reparada com fio de polipropileno 5.0 e a cavidade drenada com drenos de Penrose em ambos os flancos.O paciente apresentou rápida recuperação com alta da unidade de terapia intensiva após duas semanas de sua admissão. Sete dias após, ainda durante o seu internamento, o doente apresentou parada cardíaca súbita refratária à reanimação. A necropsia demonstrou tratar-se de uma extensa embolia pulmonar.

\section{DISCUSSÃO}

O manejo das lesões vasculares hepáticas é complexo e controverso, sendo que a maioria dos doentes evolui para óbito independente da técnica empregada para correção cirúrgica. Embora a compressão do fígado por compressas às vezes seja bem sucedida, normalmente a mesma falha quando a lesão se estende à veia cava. É sabido que a compressão por compressas de uma lesão vascular de RVC ou mesmo de veia hepática pode produzir oclusão permanente da lesão sem novo sangramento ${ }^{4}$.

O uso do SAC é extremamente controverso, apesar de relatos de sobrevida de até $33 \%$ quando utilizados precocemente $^{3}$. Porém a maioria dos Centros de Trauma não foram capazes de reproduzir estes resultados.

No caso ora apresentado descreve-se um caso de lesão grave de RVC tratado com balão intra-hepático, ao invés de compressão extrínseca ou do acesso direto e sutura. Este é o primeiro caso da literatura que se descreve o uso de balão intra-hepático no tratamento de RVC. Anteriormente o uso de balão só havia sido descrito para lesões transfixantes de fíga-

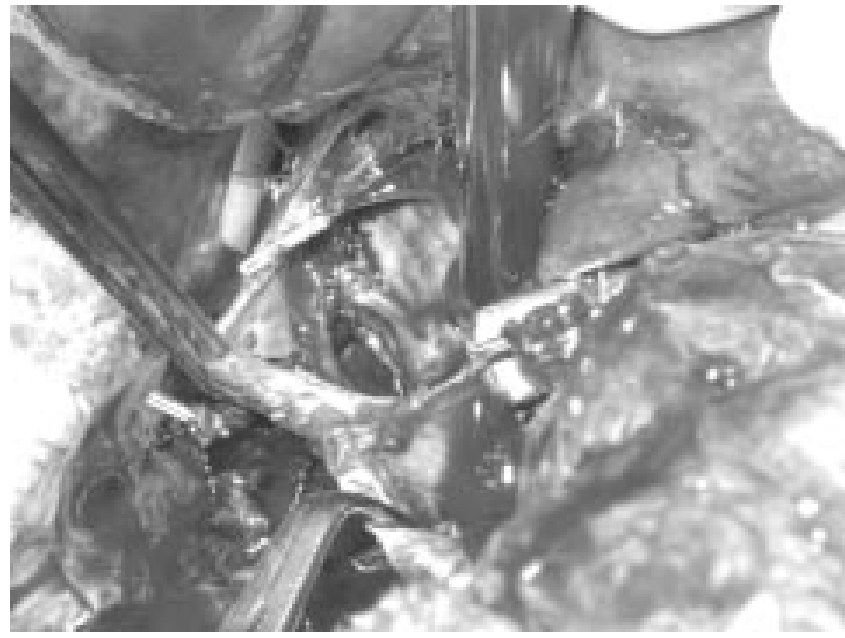

Figura 2 - Exclusão vascular do fígado com visualização de lesão transfixante de veia cava.

do ou inserido através dos vasos ilíacos como shunt ${ }^{2}$. O uso do balão foi seguramente mais simples do que instalar um SAC. Além do que permitiu ao paciente recuperar-se do trauma inicial e ser reoperado após as devidas correções da acidose, coagulopatia e hipotermia. Talvez o uso de heparina ou outro anticoagulante pudesse ter evitado a embolia pulmonar e seguramente deverá ser utilizado em um novo caso futuro.

Ao nosso ver o uso do balão intra-hepático é um recurso simples que poderia ser utilizado no manejo deste tipo de doentes, e é seguramente um recurso muito mais acessível que o SAC.

\begin{abstract}
Juxtahepatic veins, retrohepatic vena cava or major hepatic veins injuries carry a very high mortality rate regardless the technique used for its repair. Isolated survivor reports have for the most part been managed by vascular isolation techniques, usually with atriocaval shunt. We report one case with a unique management technique in a patient who sustained gunshot wound to the retrohepatic vena cava. Hemorrhage control had been obtained by intrahepatic balloon tamponade after others maneuvers have failed. The balloon was left in situ for two days as damaged control laparotomy principles and planned reoperation has been chosen (Rev. Col. Bras. Cir. 2007; 34(3): 210-211).
\end{abstract}

Key words: Venae cavae; Hemorrhage; Wounds and Injuries; Hepatic Veins.

\section{REFERÊNCIAS}

1. Pachter HL, Spencer FC, Hofstetter SR. The management of juxtahepatic venous injuries without an atriocaval shunt: preliminary clinical observations. Surgery.1986; 99(5):569-75.

2. Pachter HL, Lianf HG, Hofstetter SR. Liver and biliary tract trauma. In: Feliciano DV, Moore EE, Mattox KL, eds. Trauma. $3^{\text {rd }}$ ed. Norwalk: Appleton \& Lange; 1996. p. 508-9.

3. Walt AJ, Levison MA. Hepatic trauma: juxta-hepatic vena cava injury. In: Champion HE, Robbs JV, Trunkey DD, eds. Rob and Smith's Operative Surgery. Trauma surgery. Boston: Butterworth; 1992. p. 374-84.

4. Buckman RF, Miraliaakbari R, Badellino MM. Juxtahepatic venous injuries: a critical review of reported management strategies. J Trauma. 2000; 48(5):978-84.

5. Rotondo MF, Shwab CW, McGonigal MD, Phillips GR, Fruchterman TM, Kauder DR, Latenser BA, Angood PA.
'Damage control': an approach for improved survival in exsanguinating penetrating abdominal injury. J Trauma. 1993; 35(3):375-82; discussion 382-3.

Como citar este artigo:

Nicoluzzi JEL, Rangel M, Von Bahten LC. Uso de balão intrahepático para controle temporário da hemorragia em lesão da veia cava retro-hepática. Rev Col Bras Cir. [periódico na Internet]. 2007; 34(3). Disponível em URL: http://www.scielo.br/rcbc

Endereço para correspondência:

Dr João Eduardo Leal Nicoluzzi

Rua: Santo Amaro, 118 Água Verde.

80620-330 - Curitiba - PR

Telefone: 41-2646719

E-mail : jenicoluzz@yahoo.com 